\title{
Editoriale Capacità e possibilità di incidenza nel processo di formazione delle regole europee quale ambito di ricerca universitaria
}

\author{
Alberto Quagli*
}

È un dato di fatto che la normativa riguardante la comunicazione finanziaria delle imprese italiane discenda dalle decisioni della Commissione Europea; tali decisioni si traducono in Direttive e Regolamenti che l'Italia, come Paese membro, può solo applicare, al limite esercitando solo alcune opzioni laddove permesse dalle norme Comunitarie. Tale approccio caratterizza ormai non solo le regole applicabili alla materia del bilancio e della contabilità, ma anche alla comunicazione finanziaria di tipo non contabile delle società quotate, come informazioni da fornire circa gli assetti proprietari e di governance, operatività sui titoli, e altro ancora.

Il fenomeno in parola è stato affrontato dagli studiosi delle nostre discipline con vari atteggiamenti.

Si può adottare un atteggiamento "reazionario", intendendo il comportamento di coloro che criticano le innovazioni normative di derivazione extranazionale, proclamando la superiorità dell'approccio nazionale. Tale atteggiamento, per quanto dignitoso moralmente e intellettualmente lodevole, riveste ben scarsa utilità pratica, tenuto conto che le realtà aziendali dovranno inevitabilmente muoversi secondo le nuove regole.

Altro possibile atteggiamento è quello "passivo", che consiste nel prendere come un dato inevitabile e con un po' di fatalismo le nuove regole sovranazionali, cercando poi di capire come queste si colleghino al contesto nazionale, quali problemi risolvono e quali nuove questioni suscitano, come ad esempio è accaduto quando si sono discussi i riflessi delle regole IFRS approvate dall'Unione Europea sulla normativa fiscale italiana, profilo ancora non perfettamente chiarito a distanza di alcuni anni dalla prima applicazione.

Un terzo atteggiamento è quello "attivo", volto ad influenzare il processo di formazione delle regole nel senso che si ritiene più corretto, a sua volta

* Editor di Financial Reporting. 
discendente o da un razionale esame della questione o da una preordinata difesa nazionalistica di certi interessi particolari.

Se si dà un rapido sguardo alla produzione scientifica della nostra disciplina nel contesto nazionale, chi scrive ha l'impressione che prevalga l'atteggiamento "passivo", che rappresenta in fin dei conti la naturale predisposizione accademica tipica delle scienze sociali volta all'osservazione della realtà, piuttosto che finalizzata ad incidere su di essa. Nei migliori casi, si considera il riflesso operativo delle nuove regole, tramite analisi teoriche corredate da risultanze empiriche, cercando di evidenziare la capacità delle aziende di adattarsi al nuovo quadro e i vari riflessi in termini di comportamenti concreti indotti dalle innovazioni normative. In altri casi, ci si limita ad un commento in chiave esplicativa delle modifiche legislative, con alcune riflessioni su questioni non ancora del tutto chiare. Si tratta di lavori molto utili, sia ben chiaro, che possono favorire anche riflessioni circa modifiche normative necessarie.

L'atteggiamento "attivo" consiste invece nel proporre ai competenti organi deliberativi idee circa nuovi argomenti da discutere o risposte circa domande che tali organi periodicamente rivolgono "a tutti coloro che sono interessati" tramite processi di consultazione, per capire quale possa essere la "generale accettazione" delle nuove regole, fine ultimo al quale ogni standard riferito alla comunicazione finanziaria dovrebbe mirare. In fin dei conti, questo atteggiamento rappresenta una lecita aspettativa rivolta verso l'Accademia, ipotizzando, speriamo con buona ragione, che su certi temi siano gli accademici a "saperne di più" e, conseguentemente, a poter proporre idee migliori.

Quest'ultimo atteggiamento, invece, è decisamente più raro, per una serie di ragioni. A mio avviso le cause sono tre, tra loro correlate, e si riferiscono: 1) al modo tipico di lavorare proprio del ricercatore universitario di materie aziendali; 2) ai vantaggi personali derivabili da questo atteggiamento; 3 ) ai profili organizzativi.

Per quanto riguarda il modo di lavorare, l'adozione di un atteggiamento attivo nel senso sopra definito presuppone un lavoro collaborativo tra molti colleghi. La posizione personale in linea generale ha peso ben minore di quanto non sia un'idea o un parere, espressa da un gruppo molto più ampio di colleghi che, coeteris paribus, risulterà maggiormente rappresentativo dell' intera categoria. Nelle discipline aziendali, diversamente da quanto accade in altre branche delle scienze (si pensi ad esempio alla medicina), il lavoro di gruppo è una pratica finora meno adottata rispetto al contributo individuale. I criteri di valutazione per l'accesso alle carriere si basano sulla valutazione del singolo e a tutt'oggi rappresenta condizione sufficiente per l'esclusione di un'opera dalle valutazioni la circostanza che il "contributo individuale non sia chiaramente individuato", concetto che è la negazione stessa dell'idea di lavoro di gruppo, di progetto in comune. Si considerino, inoltre, gli aspetti pratici legati alla difficoltà di trovare momenti buoni per discutere a ranghi allargati di questioni che non possono essere risolte nello spazio di una mezza giornata. 
Per quanto riguarda i vantaggi personali derivabili dall'atteggiamento "attivo", si deve osservare che oggettivamente contributi del genere nascono per essere proposti ad organi legislativi, non per essere pubblicati su riviste scientifiche e collane editoriali di stampo accademico. Un editore ha maggiori difficoltà nel pubblicare lavori contenenti idee non immediatamente applicabili dalla pratica operante, che potrebbero sembrare anche eccessivamente sganciati dalla realtà. La possibilità che tali contributi siano considerati come risultati dell'attività scientifica, e quindi valutabili per la promozione personale, presuppone tutt'ora la "buona" collocazione editoriale, oltre al sopra ricordato profilo della possibilità di enucleare il contributo individuale.

Circa il profilo organizzativo, si può capire come un atteggiamento "attivo" presupponga anzitutto la diffusione della conoscenza circa le modalità concrete con la quali partecipare al processo, come l'esistenza di questionari aperti o di consultazioni in atto o comunque aperte. Si osserva poi che nel concreto un peso rilevante potrebbe essere ricoperto dalle associazioni accademiche, volte a promuovere queste forme di contributi. Su quest'ultimo profilo, invero si deve rilevare che tanto AIDEA quanto SIDREA stiano promuovendo questa pratica tramite la formazione di gruppi di studio, anche se finora circoscritta all'incidenza sulle sole norme "italiane". Altre aspetto rilevante, che conferisce maggiore incentivo all'adozione di un comportamento "attivo", consiste nell'esistenza di un contatto istituzionale tra il gruppo di ricercatori o della loro associazione accademiche ed altri enti e soggetti che possono, in virtù del ruolo ricoperto, sospingere "politicamente" i contributi. Quest'ultima non è una condizione necessaria, ma indubbiamente agevola il processo.

Con piccolo sforzo, un notevole incentivo verso una maggiore propensione all'esercizio del ruolo attivo potrebbe consistere nel riconoscimento di tale attività da parte delle associazioni accademiche di riferimento: considerare quale titolo valido per la valutazione dei curricula la partecipazione di un ricercatore ad un contributo propositivo nel senso sopra indicato, con le forme e le modalità che si ritengano più opportune, rappresenterebbe un sicuro motore di sviluppo per la crescente diffusione dell'atteggiamento "attivo".

Occasioni per esercitare attivamente proposte valide non mancano. Solo per citare alcuni prossime decisioni in ambito continentale di estremo rilievo per la comunicazione finanziaria delle aziende: l'Unione Europea ha in corso la rivisitazione della IV e della VII Direttiva e, contemporaneamente, sta decidendo in merito alle semplificazioni per i bilanci delle PMI (scegliendo anche se adottare o meno gli IFRS per le PMI). Cambieranno di conseguenza le norme del Codice Civile in materia di bilanci. Entro un anno, tale processo sarà presumibilmente terminato e a quel punto sarà inutile lamentarsi. È importante che le nostre idee su come dovrebbero essere i bilanci aziendali siano presentate in fretta. Ne va della nostra capacità di intervenire sulla realtà e di dimostrare che gli universitari non sono solo intellettuali ma anche persone desiderose di migliorare le condizioni di vita delle aziende. 